Revista Baiana de Saúde Pública
ARTIGO ORIGINAL

\section{SAÚDE MENTAL NA ATENÇÃO BÁSICA}

Teresa Neuma Modesto ${ }^{b}$

Darci Neves Santos

\section{Resumo}

A escassez de informações no contexto brasileiro sobre ações de Saúde Mental na Atenção Básica tem sido apontada. Este estudo teve como objetivo identificar o perfil diagnóstico e sócio-demográfico de pacientes assistidos em Saúde Mental no Programa de Saúde da Família em Cachoeira, Bahia. Realizou-se um estudo de corte transversal, examinando 100 (cem) prontuários com diagnósticos psiquiátricos atendidos em ambulatório. Encontrou-se que 54\% dos atendimentos foram do sexo masculino, sendo $67 \%$ adultos jovens, $49 \%$ de procedência urbana e 63\% sem ocupação. Dos diagnósticos encontrados 37\% foram de esquizofrenia, transtornos esquizotípicos e delirantes e 18\% de transtornos afetivos. Os achados sugerem a inclusão das ações de Saúde Mental na Atenção Básica, considerando o elevado percentual de transtornos mentais severos e a precária inserção sócio-econômica desses indivíduos no território onde vivem.

Palavras-chave: Saúde mental. Epidemiologia. Atenção básica. Reforma psiquiátrica.

\section{MENTAL HEALTH IN BASIC HEALTH CARE}

\begin{abstract}
The scarcity of information on mental health in basic health care, within the Brazilian context, has been revealed. The objective of this study was to identify the sociodemographic and diagnostic profile of patients treated for mental health in the Family Health Program in Cachoeira, Bahia. A cross-sectional study was carried out assessing 100 case records with psychiatric diagnoses treated as outpatients. The results illustrated that $54 \%$ of the patients treated were male, $67 \%$ young adults, $49 \%$ living in the urban area, and 63\% unemployed. From the diagnoses found, $37 \%$ related to schizophrenia, schizotypic and delirious disorders, and $18 \%$
\end{abstract}

a Trabalho desenvolvido a partir do Curso de Especialização em Saúde Mental/UFBA/MS/SESAB.

b Auditora do SUS/SESAB.

Endereço para correspondência: Praça Dois de Julho, 184, apto. 1801, Campo Grande, CEP 40.080-121, Salvador, Bahia. Telefones: (71) 3336-9883; (71) 8816-7875. Email: teresamodesto@hotmail.com

c Instituto de Saúde Coletiva (ISC), Universidade Federal da Bahia (UFBA). 
related to emotional disorders. The findings suggest the need to include mental health procedures in basic health care, considering the high percentage of severe mental disorders and the precarious socioeconomic insertion of these individuals in the place where they live.

Key words: Mental health. Epidemiology. Basic health care. Psychiatric reform.

\section{INTRODUÇÃO}

O processo de descentralização do Sistema Único de Saúde (SUS) exige que se empregue a lógica da integralidade, permitindo assim o entrelaçamento de diversos programas, no reconhecimento dos aspectos biológicos, psicológicos e sociais relativos à saúde da população. Nessa perspectiva, este trabalho pretende discutir aspectos da saúde mental na Atenção Básica, focalizando essa integralidade.

Sem a inserção de uma equipe mínima de Saúde Mental na Atenção Básica, o que se propõe fica dissociado. Nenhum sistema sanitário estará completo se não atender às necessidades de Saúde Mental da população.'

A reforma psiquiátrica brasileira trabalha com a lógica da desospitalização, focada na família e na comunidade, desenvolvendo ações de promoção, prevenção, tratamento e reabilitação. Esses são os mesmos princípios e diretrizes propostos na Atenção Básica², portanto, um eixo estratégico para a inserção das ações de Saúde Mental e um campo fértil para essa nova forma de pensar saúde, envolvendo uma rede de atores com saberes e fazeres diferenciados, reforçando-se assim mais um dos princípios norteadores do Sistema Único de Saúde, a intersetorialidade.

Sendo a Atenção Básica a porta de entrada do sistema de saúde ${ }^{3}$, a inserção das ações de Saúde Mental permite que os sintomas psíquicos sejam detectados e tratados precocemente, evitando-se internações desnecessárias.

Considerando a precariedade das informações sobre ações de Saúde Mental na Atenção Básica, pretende-se com este trabalho apresentar dados relativos ao perfil clínico/ epidemiológico de pacientes psiquiátricos atendidos na Atenção Básica no município de Cachoeira (BA), visando uma contribuição para a implementação das ações em Saúde Mental na Atenção Básica no Sistema Único de Saúde.

\section{MATERIAL E MÉTODOS}

Realizou-se um estudo de corte transversal da demanda de pacientes psiquiátricos assistidos no Programa de Saúde da Família, em Cachoeira (BA), no ano de 2001, examinando-se 100 prontuários médicos de indivíduos maiores de 18 anos, atendidos no segundo trimestre de 2001. Obteve-se informações sobre: idade, sexo, ocupação, encaminhamento e diagnóstico pela CID10. 
Revista Baiana de Saúde Pública

\section{RESULTADOS}

Dos 100 prontuários examinados, 54\% foram de pacientes do sexo masculino, sendo $67 \%$ de adultos jovens entre 20 e 49 anos, 49\% de procedência urbana e 63\% sem ocupação (Tabela 1). Observa-se que 38\% dos encaminhamentos para o atendimento em Saúde Mental foram realizados pelos agentes comunitários de saúde (ACS), 23\% pelos médicos do Programa de Saúde da Família e 24\% por demanda espontânea (Tabela 2). Os transtornos psicóticos graves apresentaram o maior percentual (37\%) de esquizofrenia, transtornos esquizotípicos e delirantes, seguidos de $18 \%$ para transtornos afetivos, $12 \%$ de epilepsias, 10\% de retardo mental, $8 \%$ de transtornos neuróticos, $10 \%$ de uso abusivo de álcool e 5\% sem diagnóstico preciso (Tabela 3). Chama atenção a predominância dos transtornos psicóticos graves e das epilepsias.

Tabela 1. Características sócio-demográficas dos pacientes atendidos

\begin{tabular}{|c|c|c|c|}
\hline & Dados sócio-demográficos & $n^{0}$ & $\%$ \\
\hline \multicolumn{4}{|l|}{ Sexo } \\
\hline & Masculino & 54 & 54,0 \\
\hline & Feminino & 46 & 46,0 \\
\hline Total & & 100 & 100,0 \\
\hline \multicolumn{4}{|c|}{ Faixa etária } \\
\hline & 2 a 8 & 4 & 4,0 \\
\hline & 9 a 13 & 5 & 5,0 \\
\hline & 14 a 19 & 11 & 11,0 \\
\hline & 20 a 49 & 67 & 67,0 \\
\hline & Acima de 49 & 13 & 13,0 \\
\hline Total & & 100 & 100,0 \\
\hline \multicolumn{4}{|c|}{ Procedência } \\
\hline & Urbana & 49 & 49,0 \\
\hline & Rural & 47 & 47,0 \\
\hline & Sem informação & 4 & 4,0 \\
\hline Total & & 100 & 100,0 \\
\hline \multicolumn{4}{|c|}{ Ocupação } \\
\hline & Sem ocupação & 63 & 63,0 \\
\hline & Dona de casa & 13 & 13,0 \\
\hline & Aposentado & 8 & 8,0 \\
\hline & Funcionário público & 5 & 5,0 \\
\hline & Estudante & 3 & 3,0 \\
\hline & Subempregado & 8 & 8,0 \\
\hline Total & & 100 & 100,0 \\
\hline
\end{tabular}


Tabela 2. Distribuição dos pacientes segundo fontes de encaminhamento ao psiquiatra

\begin{tabular}{l|r|r}
\hline Fontes de encaminhamento ao Psiquiatra & $\mathbf{n}^{\mathbf{0}}$ & $\mathbf{\%}$ \\
\hline ACS & 38 & 38,0 \\
Demanda espontânea & 24 & 24,0 \\
Médico PSF & 23 & 23,0 \\
Hospital psiquiátrico & 12 & 12,0 \\
Juízo da comarca & 2 & 2,0 \\
Local de trabalho & 1 & 1,0 \\
Total & $\mathbf{1 0 0}$ & $\mathbf{1 0 0 , 0}$ \\
\hline
\end{tabular}

Tabela 3. Distribuição dos pacientes por diagnóstico psiquiátrico segundo o CID 10

\begin{tabular}{lr|r}
\hline \multicolumn{1}{c|}{ Diagnóstico psiquiátrico - CID 10 } & $\mathbf{n}^{\mathbf{0}}$ & \% \\
\hline Psicoses & 37 & 37,0 \\
Transtornos afetivos & 18 & 18,0 \\
Epilepsias & 12 & 12,0 \\
Retardo mental & 10 & 10,0 \\
Neuroses & 8 & 8,0 \\
Alcoolismo & 10 & 10,0 \\
Outros & 5 & 5,0 \\
Total & $\mathbf{1 0 0}$ & $\mathbf{1 0 0 , 0}$ \\
\hline
\end{tabular}

\section{CONSIDERAÇÕES ÉTICAS}

Este trabalho foi aprovado pelo Comitê de Ética em Pesquisa do Instituto de Saúde Coletiva, tendo sido protocolado sob o número 045-05/CEP-ISC.

\section{DISCUSSÃO}

A importância de dados referentes às características sociodemográficas em trabalhos com portadores de transtornos mentais torna-se imprescindível, levando-se em conta os pressupostos teórico-práticos da reforma psiquiátrica, que recomenda o tratamento bio-psicosocial. ${ }^{4}$ Diante da amostra de $63 \%$ dos pacientes sem inserção produtiva/formal no mercado de trabalho, deduz-se o baixo grau de autonomia e dependência social, sugerindo uma decorrência da 
Revista Baiana de Saúde Pública

enfermidade psíquica. No entanto os pacientes psiquiátricos foram há décadas segregados da sociedade, internados por longos períodos de tempo em hospitais psiquiátricos, favorecendo também a desabilitação que dificulta a reinserção social/produtiva.

Observa-se uma maior percentagem de portadores de transtornos mentais dentro da faixa etária de 20 a 49 anos, o que parece estar relacionado com o período médio de instalação dos transtornos mentais, ocasião em que os efeitos dessas doenças são mais severos. ${ }^{5}$

Chama atenção a percentagem de psicoses severas que procuram os serviços da Atenção Básica. Se esses casos fossem tratados e acompanhados por especialistas nesses espaços, seria desnecessário um internamento em hospital psiquiátrico. Um achado surpreendente foi o número de pacientes com diagnóstico de epilepsia. Nos países em desenvolvimento, pela carência de serviços de Neurologia, os portadores dessa enfermidade são assistidos por psiquiatras ${ }^{6}$, mas acredita-se que cuidados especializados devam ser oferecidos, com solicitação de exames e medicações especializadas entre outros.

Constatou-se a importância da atuação dos agentes comunitários de saúde (ACS), detectando precocemente os casos e encaminhando-os para a psiquiatra.

Há muito caminho a ser percorrido na inserção das ações de Saúde Mental na Atenção Básica. O pensamento sobre esta questão deve superar os conceitos organicistas e a lógica da exclusão, exigindo tempo para mudança dos atuais conceitos e práticas. Por outro lado, os especialistas (psiquiatras) por muito tempo estiveram afastados da Atenção Básica e da Saúde Pública, criando-se assim um hiato, que não é o que se pretende. Nossos achados permitem uma caracterização inicial das ações de Saúde Mental na Atenção Básica, reforçando a necessidade de outros estudos em nosso meio para orientar a organização do cuidado especializado na Atenção Básica.

\section{REFERÊNCIAS}

1. OMS-Organização Mundial da Saúde. A introdução de um componente de Saúde Mental na Atenção Primária. Genebra; 1990.

2. Brasil. Ministério da Saúde. Oficina de Trabalho para discussão do Plano Nacional de Inclusão das Ações de Saúde Mental na Atenção Básica. Brasília; 2001.

3. Starfield B. Atenção primária. Brasília: Ministério da Saúde; 2002.

4. Amarante P. Loucos pela vida - A trajetória da reforma psiquiátrica no Brasil. Rio de Janeiro: Fundação Osvaldo Cruz; 1995. 
5. Talbott J, Hales TI, Yudofsky S. Tratado de Psiquiatria. Porto Alegre: Artes Médicas; 1992.

6. Brasil. Ministério da Saúde. Secretaria de Atenção à Saúde. Política nacional para os distúrbios neurológicos. Brasília; 2005.

Recebido em 10.10.2006 e aprovado em 14.02.2007. 